\title{
Fission-fragment detector for DANCE based on thin scintillating films
}

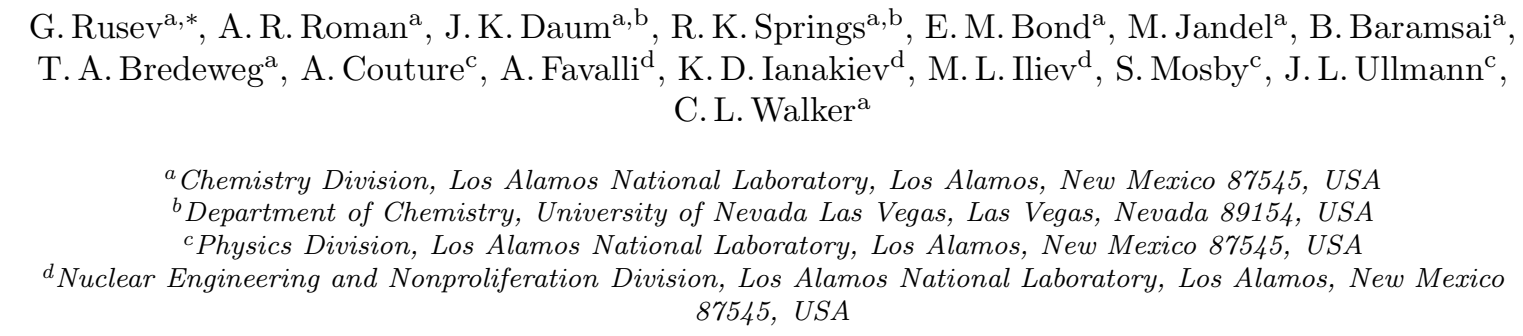

\begin{abstract}
A fission-fragment detector based on thin scintillating films has been built to serve as a trigger/veto detector in neutron-induced fission measurements at DANCE. The fissile material is surrounded by scintillating films providing $4 \pi$ detection of the fission fragments. The scintillation photons were registered with silicon photomultipliers. A measurement of the ${ }^{235} \mathrm{U}(n, f)$ reaction with this detector at DANCE revealed a correct time-of-flight spectrum and provided an estimate for the efficiency of the prototype detector of $11.6(7) \%$. Design and test measurements with the detector are described.
\end{abstract}

(c) 2015 Published by Elsevier Ltd.

Keywords: Thin scintillating films, Neutron-induced fission, Fission-fragment detector

\section{Introduction}

Nuclear fission is a phenomenon where a nucleus splits typically into two fragments accompanied with prompt emission of neutrons and $\gamma$ rays. Model description of the fission process requires measurements of correlations between all particles produced by the fission. Multidetector arrays are commonly used to simultaneously measure the emitted neutrons and $\gamma$ rays. An active target registering one or both fission fragments is often used to label the beginning of a fission event for a multidetector system. Neutron capture is a competing process to the neutron-induced fission and subject to research as well. Such an active target can be used as a trigger or a veto detector depending on the reaction studied, neutron-induced fission or neutron capture, respectively.

*Gencho Rusev, Tel.: +1 505665 7784, Email address: rusev@lanl.gov 
The $4 \pi \gamma$-ray calorimeter - the Detector for Advanced Neutron-Capture Experiments (DANCE) [1] is often used in studies of the fission process [2]. It is located at flight path 14 of the Manuel J. Lujan, Jr. Neutron Scattering Center at the Los Alamos Neutron Science Center (LANSCE). A white spectrum of neutrons is produced via spallation of a tungsten target with an $800-\mathrm{MeV}$ proton beam. The energy of the neutrons is moderated by a $2.54-\mathrm{cm}$ thick water block, increasing the flux of thermal neutrons. Fission studies at DANCE have previously been conducted with single target parallel-plate avalanche counters (PPAC) [3, 4].

In this publication, we present the design of a new trigger/veto detector for DANCE, based on thin scintillating films, as an alternative to the well established PPAC technology. The thin films detector (TFD) is reliable and easy to produce at a low cost. The fissile material is stippled onto the films (cf. Section 3), and therefore is easy to recover after the measurement. The detector does not have internal electrical connections that could fail during the measurement. Similarly to the PPAC, the TFD provides fast signals that can be used to trigger or veto DANCE events. The detector does however have a couple of disadvantages - the scintillating films contain hydrogen that scatters neutrons to DANCE and it has a non-negligible sensitivity to fast neutrons and $\gamma$ rays.

\section{Production of thin scintillating films}

The production of thin scintillating films is a critical step in building a TFD. The films have to be thin, homogeneous and without holes. Commercial scintillator paint can be used in contrast to the pioneering work done by Muga [5] on thin scintillating films. Many factors can impact the final thickness and the quality of the scintillating film. The solvent used to dilute the scintillator paint as well as the dilution of the paint are major factors. A solvent with a high vapor point will evaporate easily, causing the paint to dry quickly before it can become uniform and thin. The extent to which the paint is diluted with solvent impacts the viscosity of the paint solution and how thin the final film can be produced. Finally, the speed at which the spin coater is spun will also change the thickness - faster speeds produce thinner films. In addition, uneven speeds will give non-uniform films.

A precision spin coater $\mathrm{KW}-4 \mathrm{~A}^{1}$ was used for the uniform deposition of thin scintillating films

\footnotetext{
${ }^{1}$ Chemat Technology Inc., http://www.chemat.com/chematscientific/KW-4A.aspx/.
} 

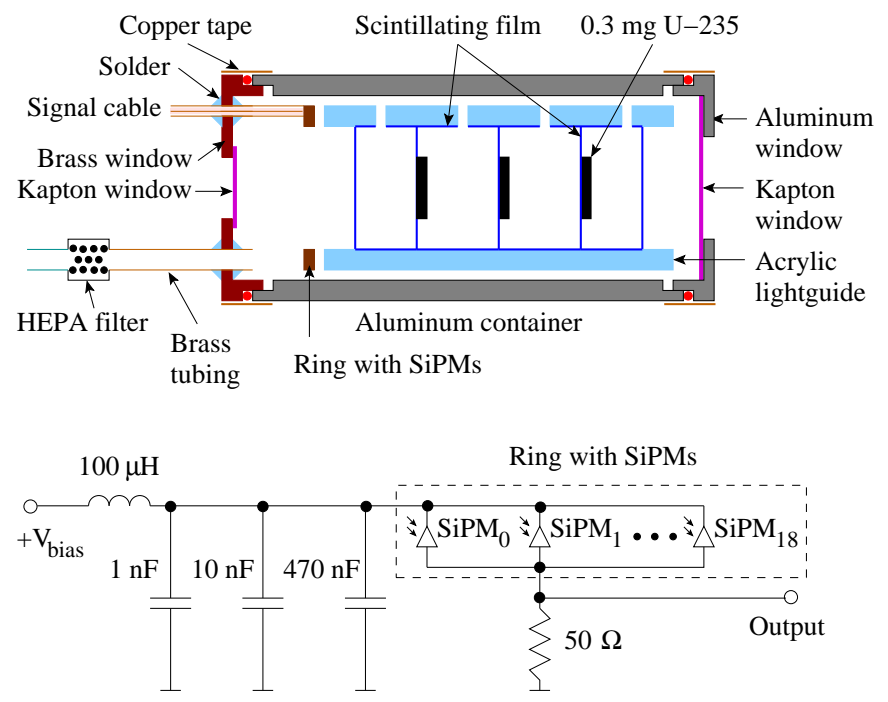

Figure 1. (Color online) Schematic drawing of the prototype TFD. The detector consists of five films, the middle three of them have small deposition of ${ }^{235} \mathrm{U}$. All films are attached to acrylic light-guide rings glued together in a solid body. The scintillations are registered by a ring of 19 SiPMs connected in parallel. The electronic scheme is shown in the bottom panel. The TFD is secured in an aluminum canister with Kapton windows to prevent contamination of the DANCE beam line. The TFD is evacuated and vented through a HEPA filter. 
onto a $7 \mu \mathrm{m}$ thick aluminized Mylar substrate. The Mylar substrate was attached to a $10 \mathrm{~cm}$ diameter silicon wafer using double-sided tape. The silicon wafer was placed in the center of the vacuum chuck of the spin coater and then the house vacuum was turned on. The spin coater was programmed to a speed of $1000 \mathrm{rpm}$ and a spin time of $50 \mathrm{sec}$. Thin scintillating films were produced by covering the Mylar substrate with plastic scintillator material. The plastic scintillator that was used to fabricate the scintillating films was the commercially available EJ- $296^{2}$ plastic scintillator paint. The scintillator paint was not diluted with solvent. A $2 \mathrm{~mL}$ aliquot of the plastic scintillator material was placed in the center of the Mylar substrate that was mounted on the spin coater using a transfer pipette as soon as the spin coater reached the desired speed. Over a period of a few days the thin scintillating films began to separate from the Mylar substrate. The film was detached from the substrate, left to dry completely and then cut to a square with area of $5 \times 5 \mathrm{~cm}^{2}$. The typical thickness of the produced films was $10-15 \mu \mathrm{m}$ considering density of $1.02 \mathrm{~g} / \mathrm{cm}^{3}$.

\section{Design of the fission-fragment detector}

The TFD has been designed to consist of several films, each containing 1-2 mg of fissile actinide material. The deposition is performed by "stippling". The actinide material is first dissolved in nitric acid and then a small drop of it is placed on the film. The films are left for a day in a fume hood until the acid evaporates. Only a spot with diameter of 2-3 mm containing the fissile material remains on the film. The stippling method creates a relatively stable deposit, but it will provide removable contamination if touched. It has to be noted that the stippling method is a low cost and easy to perform technique, but it leaves the actinide atoms bound to a few oxygen atoms and nitrate $\left(\mathrm{NO}_{3}\right)$ groups that can cause increased straggling of the fission fragments. The method of molecular plating, used to prepare the samples for the PPACs [6], has the advantage of forming uranium oxide layers that do not contain nitrite groups. For the purpose of the current TFD, stippling is an adequate method because our goal is to build a trigger/veto detector that does not have to provide spectroscopy information of the fission fragments.

The films are attached to acrylic light-guide rings. The rings are carefully polished, covered

\footnotetext{
${ }^{2}$ Eljen Technology Inc., http://www.eljentechnology.com/index.php/products/paints/84-ej-296/.
} 


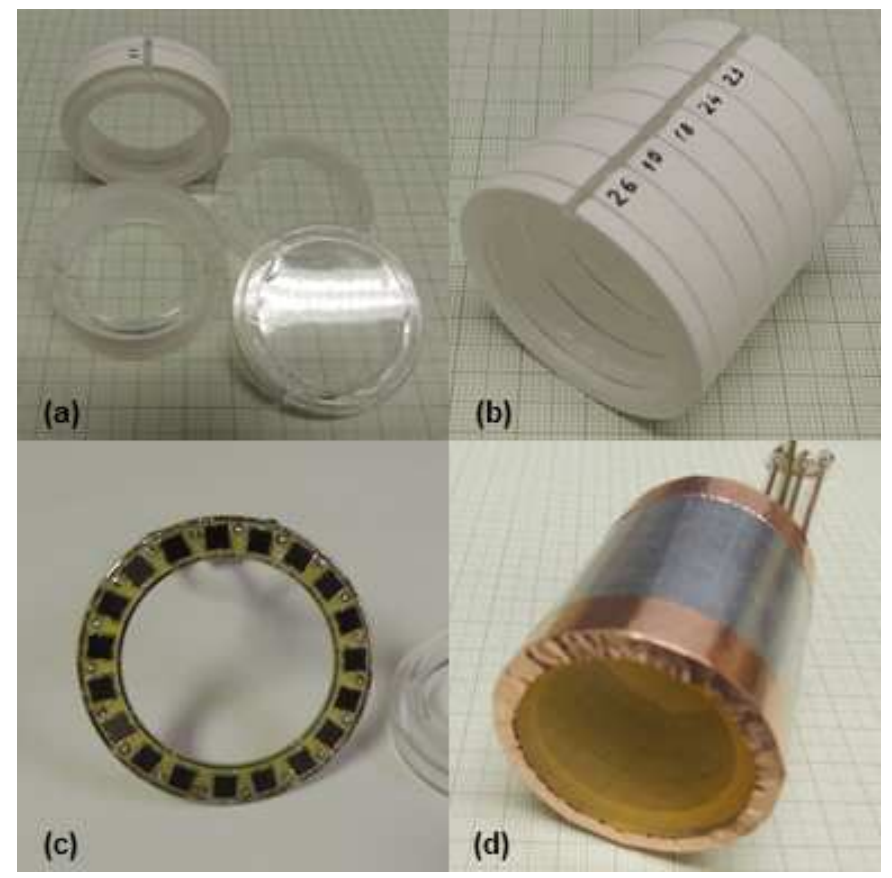

Figure 2. (Color online) Photographs of components of the prototype TFD. (a) Individual acrylic rings with thin scintillating films mounted on them. The rings are covered from outside with reflective coating and from inside with scintillator paint. (b) The complete assembly of the TFD. The notch along the light guide is for evacuating and venting the sections between the scintillating films. (c) A ring with 19 SiPMs for registering the scintillations. (d) The TFD in the aluminum canister with Kapton windows and sealed with copper tape. 
with reflective coating EJ- $510^{3}$ from the outside, and painted with the scintillator paint EJ-296 from the inside. The films are "glued" to the rings by painting the side of the ring with scintillator paint that is diluted in solvent, and attaching the film to it. Because the film is extremely thin and the paint quickly dissolves the film, the ring has to be pressed gently to the film for a moment and immediately flipped with the film-side up. The paint dries for 1-2 hours and the excessive part of the film can be cut with a scalpel. To ensure a good alignment of the fissile-material spot in the center of the ring, an alignment stand has been designed.

The rings are attached to each other with optical cement EJ-500 ${ }^{4}$. In this geometry, the fissile material is surrounded by scintillator from all sides, turning the TFD to a $4 \pi$ fission-fragment detector. The scintillations are "captured" in the light-guide body of many acrylic rings and detected by 19 silicon photomultipliers (SiPMs) MicroFC-30020-SMT ${ }^{5}$. The SiPMs are attached to the light guide by optical grease EJ-550 .

Because the TFD is meant to stay in the evacuated beam pipe of DANCE, small holes have been drilled across each of the acrylic rings for venting. The detector was placed in an aluminum canister with Kapton windows. Evacuating and venting of the detector was performed through a thin pipe ending with a HEPA filter to keep possible contamination inside the canister. A drawing of the TFD and photographs of some of the parts of the TFD are shown in Figs. 1 and 2.

\section{Performance of the fission-fragment detector}

A TFD with $0.9 \mathrm{mg}$ of ${ }^{235} \mathrm{U}$ has been tested at DANCE. The uranium was enriched to ${ }^{235} \mathrm{U}$ to 99.9\%. The fissile material was distributed over three films. An additional two blank films were used as a first and last film of the detector, cf. Fig. 1. A low-pass filter has been installed to remove the noise collected by the power line. A bias voltage of $28.5 \mathrm{~V}$ has been applied to the SiPMs. The

\footnotetext{
${ }^{3}$ Eljen Technology Inc., 

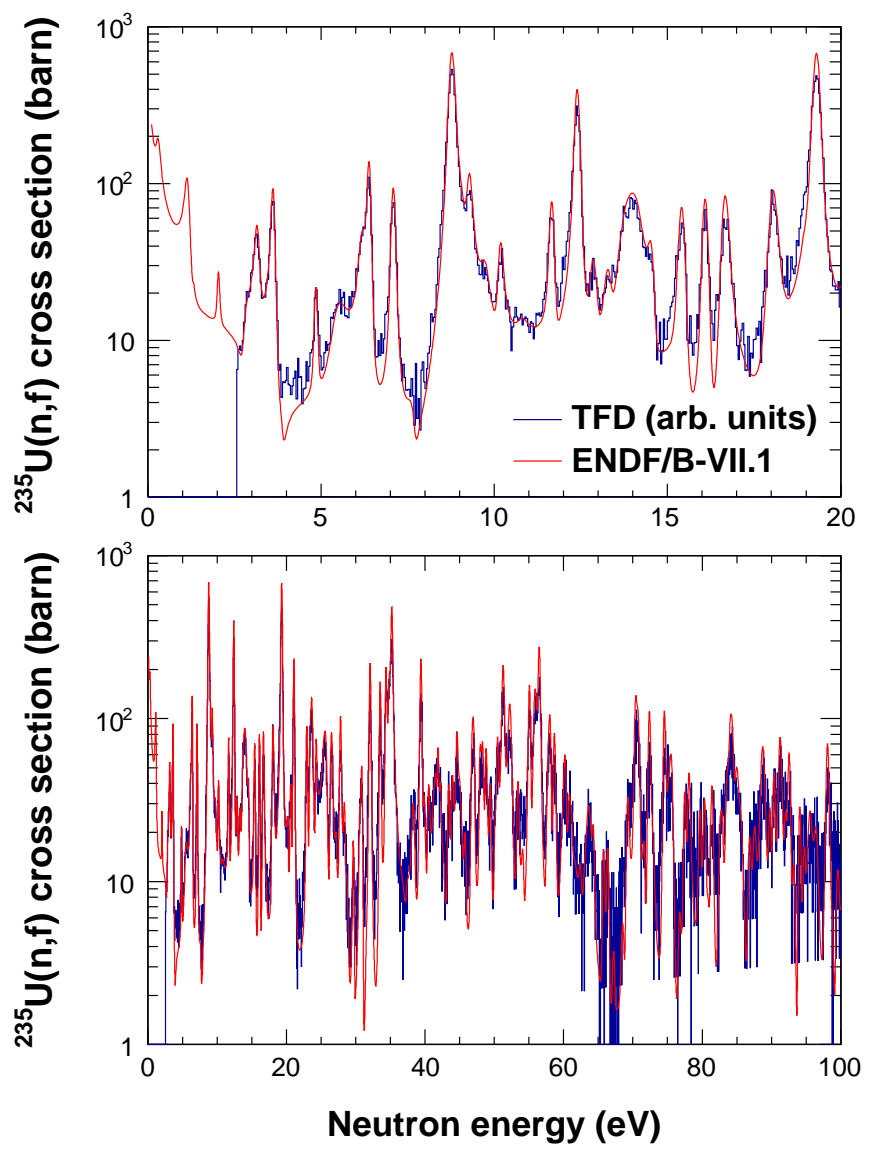

Figure 3. (Color online) A TOF spectrum measured with the TFD is compared with the ${ }^{235} \mathrm{U}(n, f)$ cross section from ENDF/B-VII.1. The raw TFD spectrum was divided by the $1 / E_{n}$ dependence of neutron flux at flight path 14 at Lujan Center to have units of cross section. It has been scaled to the ENDF data by a constant factor. The two panels show the same comparison, but for different neutron-energy ranges. 

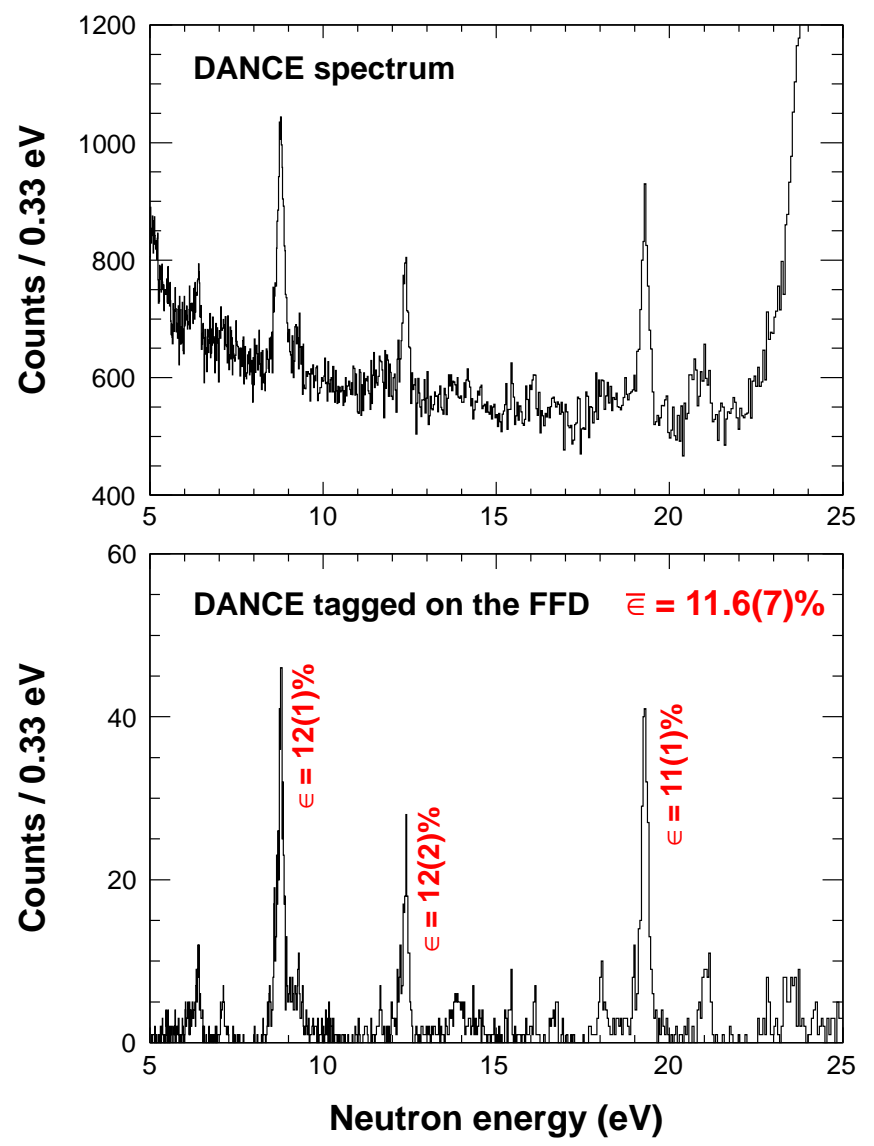

Figure 4. (Color online) TOF spectra measured with DANCE for $\gamma$-ray multiplicity 4 events (top panel) and tagged (bottom panel) on the TFD detector. The efficiency of the TFD has been obtained for three of the strong ${ }^{235} \mathrm{U}(n, f)$ resonances as a ratio of the peak areas from the two spectra. The uncertainties are only statistical. The weightedaverage value of the detection efficiency is $11.6(7) \%$. 
signals from the detector were amplified outside the DANCE cave with an Ortec $474^{7}$ timing-filter amplifier using $20 \mathrm{~ns}$ differentiation constant to restore the baseline. Then the signal was sent to one of the inputs of the $\mathrm{VX} 1730 \mathrm{~B}^{8}$ digitizers of the DANCE data-acquisition system.

A time-of-flight (TOF) spectrum was collected from the time difference between the proton-beam pick-off signal and the TFD. The TOF spectrum is compared with the ${ }^{235} \mathrm{U}(n, f)$ cross section from the evaluation ENDF/B-VII.1 [7] in Fig. 3. The counts from the TFD were converted to units of cross section by dividing the spectrum with $1 / E_{n}$ which is the shape of the neutron flux at flight path 14, cf. Fig. 8 in Ref. [8]. Because of the similar slope of the two spectra (cf. Fig. 3), the efficiency of the TFD is assumed to be constant. Deviations of the cross section obtained with the TFD from the ENDF evaluation are result of inaccurate transformation of time-of-flight to incident-neutron energy which we have used in this analysis. The transformation does not include the broadening of the neutron flux by the water moderator and other components of the neutron-production target at Lujan Center.

An estimate of the efficiency of the detector can be obtained by comparing the DANCE TOF spectrum containing all events with the one tagged on the TFD. The peak areas of the ${ }^{235} \mathrm{U}(n, f)$ resonances are proportional to the DANCE efficiency only, while the ones tagged on the TFD are proportional to the product of the DANCE and TFD efficiencies. The ratio of the two areas will give us the efficiency of the TFD with uncertainty completely statistical. The two spectra are shown in Fig. 4. A weighted average of the three strong resonances gives a value of $11.6(7) \%$ for the TFD efficiency.

Sensitivity of the detector to $\gamma$ rays and neutrons was studied with a ${ }^{252} \mathrm{Cf}$ source with neutronemission timing [9] with $\alpha$ activity of $30.3 \mathrm{kBq}$. A blank detector without any ${ }^{235} \mathrm{U}$ deposition was placed in a piece of beam pipe. The pipe ended with a break-out box with feedthroughs for powering the detector and taking the signals out. The other end of the pipe was covered with a thin aluminum foil for light insulation and behind it the ${ }^{252} \mathrm{Cf}$ source was positioned. The distance between the source and the middle of the detector was $50 \mathrm{~cm}$. A TOF spectrum, shown in Fig. 5, has been measured for 32.6 days. Integrating the neutron peak in the TOF spectrum and considering the solid angle of the detector, the activity of the source and the measurement time we calculated an

\footnotetext{
${ }^{7}$ ORTEC, www.ortec-online.com/download/474.pdf

${ }^{8} \mathrm{CAEN}$, http://www.caen.it/jsp/Template2/CaenProd.jsp?parent=11\&idmod=779/.
} 


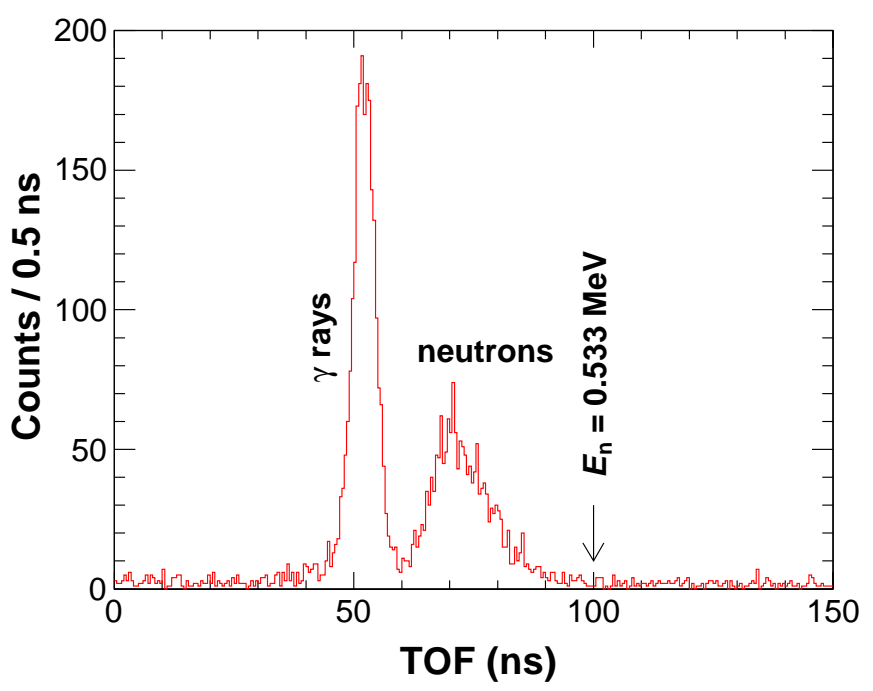

Figure 5. (Color online) A TOF spectrum measured with a blank TFD and a ${ }^{252} \mathrm{Cf}$ source with neutron-emission timing. The spectrum demonstrates that the TFD is sensitive only to $\gamma$ rays and fast neutrons, that will not affect the measurements at DANCE. Example neutron energy is shown on the figure with an arrow.

efficiency of $0.03 \%$ for detecting neutrons with energies above $0.5 \mathrm{MeV}$. The efficiency decreases for lower energy neutrons and is 0 for neutrons with energies below $0.5 \mathrm{MeV}$. The $\gamma$ ray and neutron sensitivity of the detector will not influence measurements at DANCE because the neutron flux at flight path 14 is negligible for neutron energies above $0.5 \mathrm{MeV}$ and measurements cannot be performed for these energies.

\section{Summary}

A fission-fragment detector based on thin scintillating films has been designed as an alternative trigger/veto detector to the PPAC for use with the DANCE array. Two TFDs have been built and tested in measurements at DANCE, one of the TFDs contained $0.9 \mathrm{mg}$ of ${ }^{235} \mathrm{U}$, while the other TFD was blank. The TFD provided a TOF spectrum from the ${ }^{235} \mathrm{U}(n, f)$ reaction very similar to the ENDF/B-VII.1 evaluation. The detection efficiency has been estimated to be 11.6(7)\%, it will be a subject of further improvements as using more efficient SiPMs and preamplifier attached at the ring printed circuit board. A bench-top measurement with a ${ }^{252} \mathrm{Cf}$ source and a blank TFD revealed that the small sensitivity of the detector to $\gamma$ rays and fast neutrons will not affect future measurements at DANCE. 
The TFD exhibits a number of advantages in comparison with the PPAC. It is relatively easier to build at smaller cost than a PPAC detector. The fissile material can be stippled onto the scintillating films, a much simpler method than the molecular plating used for the PPAC samples, and the material can be easily recovered after the measurement. The TFD does not need high electric fields, electrical connections and is free of possible electrical sparking and damages of the detector typical for the PPAC. The only electrical connections are between the ring with SiPMs and the signal and power cables. These connections can be tested in a lab prior to encapsulate the TFD in the aluminum canister.

A disadvantage of the TFD is the hydrogen contained in the films and the scattering of the neutron beam from it. The scattered neutrons could cause signals in the DANCE $\mathrm{BaF}_{2}$ detectors if they are captured in any of the barium isotopes. These DANCE events will be filtered out only if the TFD is used as a "trigger" detector. If the TFD is used as a "veto" detector then an additional measurement with an identical blank TFD is required to estimate the neutron-scattering background in the DANCE events.

\section{Acknowledgments}

This work has been supported by the U.S. Department of Energy through the LANL/LDRD Program and the U.S. Department of Energy, Office of Science, Nuclear Physics under Early Career Award No. LANL20135009.

This work benefited from the use of the LANSCE facility at the Los Alamos National Laboratory. This work was performed under the auspices of the US Department of Energy by Los Alamos National Security, LLC, under Contract No. DE-AC52-06NA25396.

J. K. D. and R. K. S. acknowledge the support of the U.S. Department of Energy through the LANL/LDRD Program, the Department of Homeland Security and the Glenn T. Seaborg Institute for this work.

\section{References}

[1] M. Heil, R. Reifarth, M. M. Fowler, et al., Nucl. Instrum. Methods A 459 (2001) 229.

[2] M. Jandel, G. Rusev, E. M. Bond, et al., Physics Procedia 59 (2014) 101. 
[3] T. A. Bredeweg, M. M. Fowler, J. A. Becker, et al., Nucl. Instrum. Methods B 261 (2007) 986.

[4] C. Y. Wu, A. Chyzh, E. Kwan, et al., Nucl. Instrum. Methods A 694 (2012) 78.

[5] M. L. Muga, D. J. Burnsed, W. E. Steeger, Nucl. Instrum. Methods 104 (1972) 605.

[6] E. M. Bond, T. A. Bredeweg, J. R. FitzPatrick, et al., J. Radioanal. Nucl. Chem. 282 (2009)

[9] G. Rusev, B. Baramsai, E. M. Bond, M. Jandel, Nucl. Instrum. Methods A 0 (2015) 0 - submitted. 